\title{
Cross-Modal Generalization: Learning in Low Resource Modalities via Meta-Alignment
}

\author{
Paul Pu Liang* \\ pliang@cs.cmu.com \\ Carnegie Mellon University \\ Pittsburgh, PA, USA
}

\author{
Peter $\mathrm{Wu}^{*}$ \\ peterw1@andrew.cmu.com \\ Carnegie Mellon University \\ Pittsburgh, PA, USA
}

\author{
Liu Ziyin \\ zliu@cat.phys.s.u-tokyo.ac.jp \\ University of Tokyo \\ Tokyo, Japan
}

\author{
Louis-Philippe Morency \\ morency@cs.cmu.com \\ Carnegie Mellon University \\ Pittsburgh, PA, USA
}

\author{
Ruslan Salakhutdinov \\ rsalakhu@cs.cmu.com \\ Carnegie Mellon University \\ Pittsburgh, PA, USA
}

\begin{abstract}
How can we generalize to a new prediction task at test time when it also uses a new modality as input? More importantly, how can we do this with as little annotated data as possible? This problem of cross-modal generalization is a new research milestone with concrete impact on real-world applications. For example, can an AI system start understanding spoken language from mostly written text? Or can it learn the visual steps of a new recipe from only text descriptions? In this work, we formalize cross-modal generalization as a learning paradigm to train a model that can (1) quickly perform new tasks (from new domains) while (2) being originally trained on a different input modality. Such a learning paradigm is crucial for generalization to low-resource modalities such as spoken speech in rare languages while utilizing a different high-resource modality such as text. One key technical challenge that makes it different from other learning paradigms such as meta-learning and domain adaptation is the presence of different source and target modalities which will require different encoders. We propose an effective solution based on meta-alignment, a novel method to align representation spaces using strongly and weakly paired cross-modal data while ensuring quick generalization to new tasks across different modalities. This approach uses key ideas from cross-modal learning and meta-learning, and presents strong results on the cross-modal generalization problem. We benchmark several approaches on 3 real-world classification tasks: few-shot recipe classification from text to images of recipes, object classification from images to audio of objects, and language classification from text to spoken speech across 100 languages spanning many rare languages. Our results demonstrate strong performance even when the new target modality has only a few (1-10) labeled samples and in the presence of noisy labels, a scenario particularly prevalent in low-resource modalities.
\end{abstract}

${ }^{*}$ Both authors contributed equally to this research.

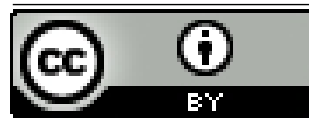

This work is licensed under a Creative Commons Attribution International 4.0 License.

MM '21, October 20-24, 2021, Virtual Event, China

(C) 2021 Copyright held by the owner/author(s).

ACM ISBN 978-1-4503-8651-7/21/10.

https://doi.org/10.1145/3474085.3475247

\section{CCS CONCEPTS}

- Information systems $\rightarrow$ Multimedia information systems; • Computing methodologies $\rightarrow$ Machine learning; Natural language processing; Speech recognition; Computer vision.

\section{KEYWORDS}

Multimodal learning, Meta-learning, Cross-modal alignment, Crossmodal retrieval

\section{ACM Reference Format:}

Paul Pu Liang, Peter Wu, Liu Ziyin, Louis-Philippe Morency, and Ruslan Salakhutdinov. 2021. Cross-Modal Generalization: Learning in Low Resource Modalities via Meta-Alignment. In Proceedings of the 29th ACM International Conference on Multimedia (MM '21), October 20-24, 2021, Virtual Event, China. ACM, New York, NY, USA, 17 pages. https://doi.org/10.1145/3474085.3475247

\section{INTRODUCTION}

One of the hallmarks of human intelligence is the ability to generalize seamlessly across heterogeneous sensory inputs and different cognitive tasks [9]. We see objects, hear sounds, feel textures, smell odors, and taste flavors to learn underlying concepts present in our world [4]. Much of AI's existing progress in multimodal learning, however, focuses primarily on a fixed predefined set of input modalities and tasks $[34,40]$ that are consistent between training and testing. As a result, it is unclear how to transfer knowledge from models trained for one modality (e.g. visual source modality) to another (e.g. audio target modality) at test time. Solving this problem is particularly important for low-resource modalities where labeled data is scarce (e.g. audio from low-resource languages [38], real-world environments [46], and medical images [14]).

As a motivating example, Figure 1 illustrates a scenario where large-scale image classification benchmarks can help audio classification, which is a less studied problem with fewer large-scale benchmarks. In this problem, a key research question becomes: how can we obtain generalization across modalities despite using separate encoders for different source (image) and target (audio) modalities? The technical challenge involves aligning shared knowledge learned from source image tasks with target audio tasks. Our problem statement differs from conventional meta-learning [17], few-shot learning [8], and domain adaptation [59] where the same source and target modality with shared encoders helps generalization by having the same representation space. 


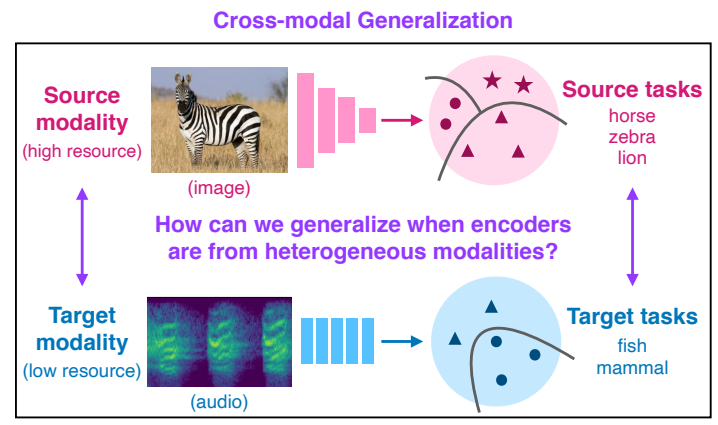

Figure 1: The cross-modal generalization paradigm brings discrepancies in both input and output spaces with new tasks expressed in new modalities. This raises a fundamental question: how can we generalize across modalities despite using separate encoders for different source (image) and target (audio) modalities? We study the minimal supervision required to perform this alignment and succeed in crossmodal generalization.

We formally define the cross-modal generalization setting as a learning paradigm to train a model that can (1) quickly perform new tasks in a target modality (i.e. meta-learning) and (2) doing so while being trained on a different source modality. Cross-modal generalization will enable the model to utilize a completely different high-resource modality during training to help low-resource modalities at test time. For example, high-resource written text can help interpret spoken speech even with low-resource data. In this paper, we formalize the conditions required for successful generalization and attempt to understand the minimal extra supervision required under partial observability across modalities and tasks. Supervision comes in the form of cross-modal meta-alignment (Figure 2) to capture a space where representations of similar concepts in different modalities are close together while ensuring quick generalization to new tasks (i.e. with just a few labels in the target modality). We introduce an algorithm called CRoMA (Cross-modal Meta-Alignment) that leverages readily available multimodal data from the internet (e.g. [34, 48, 67]) for alignment and enables accurate cross-modal generalization. Crucially, these pairings between source and target modalities may be easier to obtain than directly collecting and labeling data in the target modality. We study our proposed algorithm with both strongly and weakly paired multimodal data, showing that cross-modal generalization is possible even with limited extra supervision.

We present experiments on three real-world cross-modal tasks: (1) few-shot recipe classification from text to images of recipes, (2) object classification from images to audio of objects, and (3) language classification from text to spoken speech across 100 languages spanning many rare languages. In all cases, the goal is to classify data from a new target modality given only a few (1-10) labeled samples. We perform extensive comparisons with related approaches including target modality meta-learning and domain adaptation that would be expected to perform well since they have seen thousands of labeled examples from the target modality during meta-training. Surprisingly, CroMA is competitive with these baselines and significantly outperforms other cross-modal approaches.

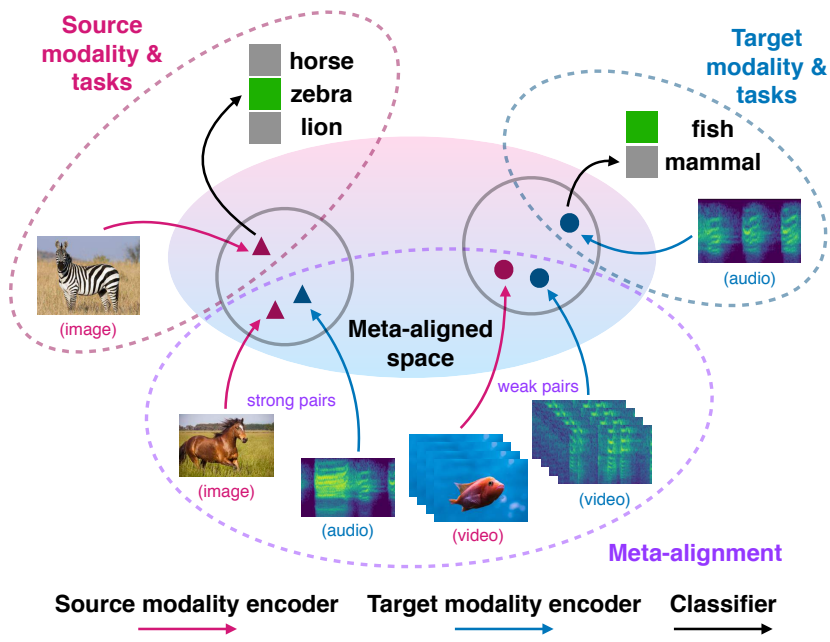

Figure 2: Meta-alignment aligns representation spaces while ensuring quick generalization to new tasks using strongly (image and audio) and weakly (video) paired data. This enables generalization to the target (audio) having only seen labeled data in the source (image), as assessed by few-shot classification and alignment tasks.

In addition, we study settings where the target modality suffers from noisy or limited data, a scenario particularly prevalent in the real world [22].

\section{RELATED WORK}

Few-shot learning has enabled strong performance from limited labeled data $[8,20,22]$ using techniques spanning data augmentation [2], metric learning [55, 61], and learning better initializations $[44,51]$. In the latter, meta-learning has emerged as a popular choice due to its simplicity using gradient-based methods [17].

Transfer learning focuses on transferring knowledge from external data (e.g. larger datasets [29], unlabeled data [12], and knowledge bases [32]) to downstream tasks where labeled data is expensive [57]. Domain adaptation similarly focuses on changing data distributions [13, 27]. However, existing works focus on data within the same modality (i.e. image domain adaptation [59], language transfer learning [12]) which simplifies alignment.

Cross-modal alignment involves learning a joint space where the representations of the same concepts expressed in different modalities are close together [4]. Alignment is useful for crossmodal retrieval (e.g. retrieving captions from images) [18] and cross-modal (or cross-lingual) representation learning [54, 63]. Several objective functions for learning aligned spaces from varying quantities of paired $[6,16,28]$ and unpaired [21] data have been proposed. However, cross-modal generalization is harder since: (1) one has to learn not just the associations between modalities but also associations to labels, (2) there is weak supervision in both modality and label space (see Table 1), (3) tasks in different modalities have different (but related) label spaces, and (4) new tasks in the target modality have to be learned using only a few samples.

Cross-modal learning: Recent work has explored knowledge transfer across modalities such as cross-modal data programming [14], cross-modal transfer learning $[28,65]$, or cross-modal few-shot 
Table 1: Data requirements for generalization (i.e. (meta)test) in a target modality where both data and labels are rare. Cross-modal generalization leverages data from abundant source modalities for low-resource target modalities, requiring only a few samples and no labels in the target beyond those used for few-shotfine-tuning.

\begin{tabular}{l||cc}
\hline \multirow{2}{*}{ APPROACHES } & \multicolumn{3}{|c}{ (META-)TRAIN } \\
& Modality & Data Labels \\
\hline Transfer learning [3] & Target & Many None \\
\hline Unsupervised pre-training [12] & Target & Many None \\
Unsupervised meta-learning [26] & Target & Many None \\
\hline Domain adaptation [59] & Target & Many Many \\
Few-shot learning [17] & Target & Many Many \\
\hline Within modality + cross-modal & Source & Many None \\
learning [14, 56, 58, 64, 66] & Target & Many Many \\
\hline \multirow{2}{*}{ Cross-modal generalization (ours) } & Source & Many Many \\
& Target & Few None \\
\hline
\end{tabular}

learning $[56,58,64,66]$. However, these all require labeled data from the target modality during meta-training (from a different domain). We study cross-modal generalization which do not assume any labeled data in the target except during few-shot classification.

\section{CROSS-MODAL GENERALIZATION}

Cross-modal generalization is a learning paradigm to quickly perform new tasks in a target modality despite being trained on a different source modality. To formalize this paradigm, we build on the definition of meta-learning [25] and generalize it to study multiple input modalities. Meta-learning uses labeled data for existing source tasks to enable fast learning on new target tasks [33]. We start by defining $M$ different heterogeneous input spaces (modalities) and $N$ different label spaces (tasks). We denote a modality by an index $m \in\{1, \ldots, M\}$ and a task by $n \in\{1, \ldots, N\}$.

Each classification problem $\mathcal{T}(m, n)$ is defined as a triplet with a modality, task, plus a joint distribution: $\mathcal{T}(m, n)=\left(\mathcal{X}_{m}, \mathcal{Y}_{n}, p_{m, n}(x, y)\right)$ $\mathcal{X}_{m}$ denotes the input space and $\mathcal{Y}_{n}$ the label space sampled from a distribution $p(m, n):=p\left(\mathcal{X}_{m}, \mathcal{Y}_{n}\right)$ given by a marginal over the entire meta-distribution, $p\left(x_{1}, \ldots, x_{M}, y_{1}, \ldots y_{N}, \mathcal{X}_{m_{1}}, \ldots \mathcal{X}_{m_{M}}, \mathcal{Y}_{n_{1}}, \ldots \mathcal{Y}_{n_{N}}\right)$. The meta-distribution gives the underlying relationships between all modalities and tasks through a hierarchical generative process $m_{i} \sim p(m), n_{j} \sim p(n)$ :fi rst picking a modality and task $\left(m_{i}, n_{j}\right)$ from priors $p(m)$ and $p(n)$ over input and output spaces, before drawing data $x_{i}$ from $\mathcal{X}_{m_{i}}$ and labels $y_{j}$ from $\mathcal{Y}_{n_{j}}$. Within each classification problem is also an underlying pairing function mapping inputs to labels through $p_{m, n}(x, y):=p(x, y \mid m, n)$ for all $x \in \mathcal{X}_{m}, y \in \mathcal{Y}_{n}$ representing the true data labeling process. To account for generalization over modalities and tasks, cross-modal generalization involves learning a single function $f_{w}$ with parameters $w$ over the meta-distribution with the following objective:

Definition1. The cross-modal generalization problem is

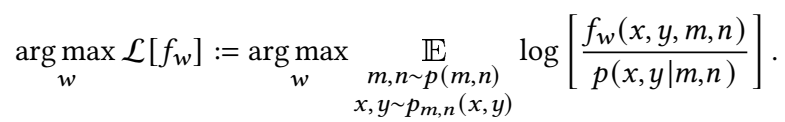

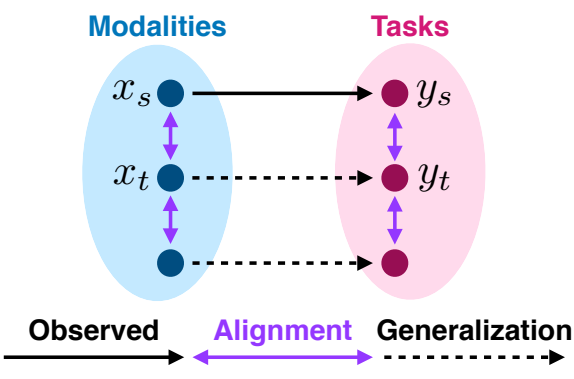

Figure 3: Observed edges are obtained via labeled datasets for source modalities $x_{s}$ and tasks $y_{s}$ (solid edges). Generalizing to target modalities $x_{t}$ and tasks $y_{t}$ (dotted edge) requires bridging modalities and tasks through alignment (purple).

In practice, the space between modalities and tasks is only partially observed: $p(x, y \mid m, n)$ is only observed for certain modalities and tasks (e.g. labeled classification tasks for images [11], or paired data across image, text, and audio in online videos [1]). For other modality-task pairs, we can only obtain inaccurate estimates $q(x, y \mid m, n)$, often due to having only limited labeled data. It is helpful to think about this partial observability as a bipartite graph $G=\left(V_{x}, V_{y}, E\right)$ between a modality set $V_{x}$ and task set $V_{y}$ (see Figure 3). A solid directed edge from $u \in V_{x}$ to $v \in V_{y}$ represents learning a classifier from modality $u$ for task $v$ given an abundance of observed labeled data, which incurs negligible generalization error. Since it is unlikely for all edges between $V_{x}$ and $V_{y}$ to exist, define the low-resource subset $\mathcal{M}$ as the complement of $E$ in $V_{x} \times V_{y}$. $\mathcal{M}$ represents the set of low-resource modalities and tasks where it is difficult to obtain labeled data. The focus of cross-modal generalization is to learn a classifier in $\mathcal{M}$ as denoted by a dashed edge. In contrast to solid edges, the lack of data in $\mathcal{M}$ incurs large error if direct supervised learning is used on dashed edges.

Therefore, the challenge in cross-modal generalization amounts tofi nding the path of lowest cumulative error between an input target modality $x_{t} \in V_{x}$ and output task $y_{t} \in V_{y}$ in $\mathcal{M}$. The key insight is to leverage cross-modal information to "bridge" modalities that are each labeled for only a subset of tasks (see purple edges in Figure 3). We model cross-modal information as $p\left(x_{s}, x_{t}\right)$, i.e. alignment between modalities $x_{s}$ and $x_{t}$, where $x_{s}$ is a source modality with high-resource data and labels $\left(x_{s}, y_{s}\right)$. When there is an abundance of paired data $\left(x_{s}, x_{t}\right)$ (solid purple edge), we say that strong alignment exists; otherwise, only weak alignment exits. Since strong alignment incurs negligible error in estimating $p\left(x_{s}, x_{t}\right)$, the alternative cross-modal path $P=\left\{\left(x_{t}, x_{s}\right),\left(x_{s}, y_{s}\right),\left(y_{s}, y_{t}\right)\right\}$ might link $x_{t}$ and $y_{t}$ with lower weighted error and is preferable to direct low-resource training for the dashed edge $\left(x_{t}, y_{t}\right)$. When only weak alignment is available, a trade-off emerges and one has to choose between the error induced by direct low-resource training and the error induced by weak alignment. $\left(y_{s}, y_{t}\right)$ models relationships across source and target tasks using approaches such as multi-task [7] or meta-learning [17]. More formally,

Definition2. Let $p\left(x_{i}, x_{j}\right)$ be known for $x_{i} \in \mathcal{D}_{m_{i}}^{x}, x_{j} \in \mathcal{D}_{m_{j}}^{x}$ and $i \neq j$. If both $p\left(x_{i} \mid x_{j}\right)$ and $p\left(x_{j} \mid x_{i}\right)$ are delta distributions (i.e., 


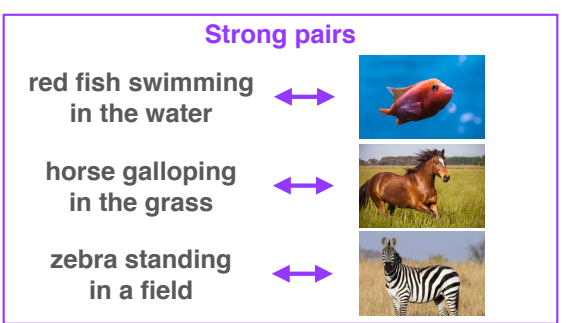

(a)

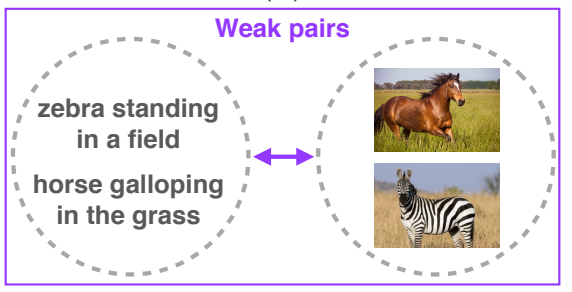

(b)

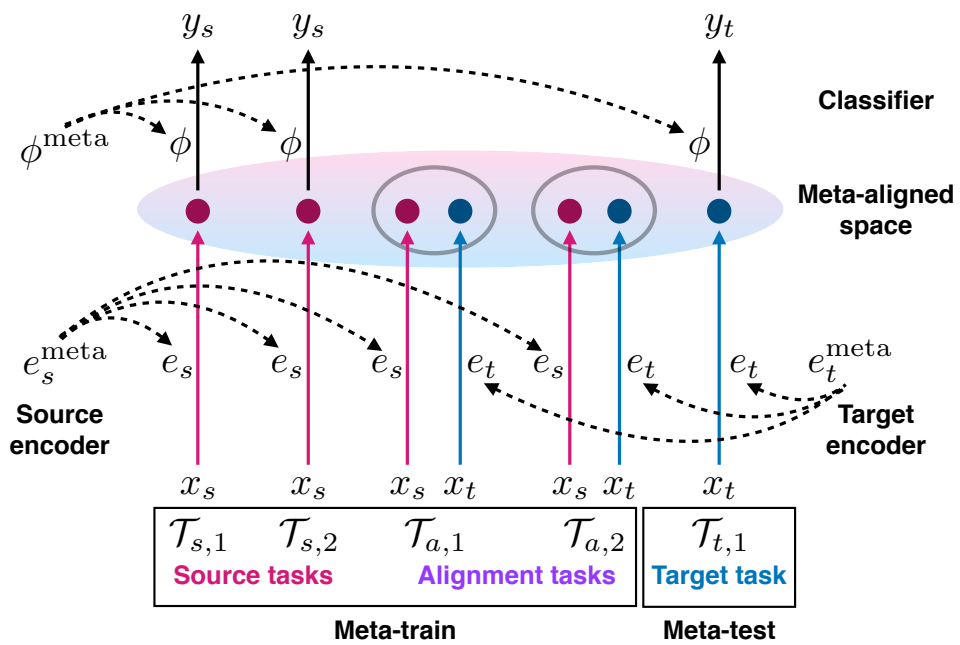

(c)

Figure 4: While strong pairs (a) provide exact, one-to-one correspondences across modalities, weak pairs (b) represent coarse semantic groupings which better reflect many-to-many cross-modal mappings and leverage weakly paired multimodal data available on the internet (e.g. videos, image captions). (c) During meta-training, meta-parameters $e_{s}^{\text {meta }}, e_{t}^{\text {meta }}, \phi^{\text {meta }}$ are trained using source modality classification tasks $\mathcal{T}_{s}$ and alignment tasks $\mathcal{T}_{a}$. Meta-testing uses trained meta-parameters for few-shot generalization to target modality tasks $\mathcal{T}_{t}$.

one-to-one mapping between $x_{i}$ and $x_{j}$ ), there is a strong alignment between modality $m_{i}$ and $m_{j}$. Otherwise, there is only weak alignment.

We now show that strong alignment can achieve optimal generalization error for tasks in the low-resource set $\mathcal{M}$.

Proposition1. (Benefit of strong alignment). Let all the modalities be pairwise strongly-aligned, then we can define a surrogate loss function $\tilde{\mathcal{L}}\left[f_{w}\right]$ such that $\mathcal{L}\left[\arg \max _{w} \tilde{\mathcal{L}}\left[f_{w}\right]\right]=0$.

The proof is provided in Appendix A. On the other hand, weak alignment may not provide perfect generalization and we elucidate some conditions when it works in Section 5.5.

In the following section, we explain an algorithm based on contrastive learning [18] to estimate $p\left(x_{s}, x_{t}\right)$ and meta-learning to model $\left(y_{s}, y_{t}\right)$, thereby achieving cross-modal generalization.

\section{CROSS-MODAL META-ALIGNMENT}

Based on our theoretical insights, we propose a practical algorithm involving two ideas: (1) learning a multimodal space via metaalignment to model cross-modal information $\left(x_{s}, x_{t}\right)(\S 4.1)$, and (2) learning a cross-modal classifier to model $\left(y_{s}, y_{t}\right)(\S 4.2)$, which jointly enable generalization to new modalities and tasks. We call our method CROMA, short for Cross-modal Meta-Alignment.

\subsection{Meta-Alignment}

Wefi rst simplify the problem by assuming access to strong pairs across modalities of the form $\left(x_{s}, x_{t}\right)$ to learn strong alignment (see Figure 4(a)). At the same time, this is not an excessively strong assumption: many multimodal datasets contain paired multimodal data (e.g. activity recognition from audio and video [1] and emotion recognition from text, speech, and gestures [41, 67]).
4.1.1 Strong alignment. In practice, given strongly aligned data, we model alignment by learning $p_{\theta}\left(x_{t} \mid x_{s}\right)$. However, directly learning a translation model $p_{\theta}\left(x_{t} \mid x_{s}\right)$ via MLE by mapping each $x_{s}$ to its corresponding $x_{t}$ is hard since $x_{s}$ and $x_{t}$ are high-dimensional and heterogeneous data sources which makes reconstruction difficult [37] (in practice, $x_{s}$ and $x_{t}$ could be extracted features but are still very high-dimensional). Instead, we use Noise Contrastive Estimation (NCE) which learns a binary classifier to distinguish paired samples $\left(x_{s}, x_{t}\right)$ from unpaired negative samples $x_{t, \text { neg, which in }}$ the asymptotic limit is an unbiased estimator of $p\left(x_{t} \mid x_{s}\right)$ [15] but is much easier in practice than generating raw data.

However, NCE does not handle new tasks at test time. We propose meta-alignment to capture an aligned space (i.e. $\left.\left(x_{s}, x_{t}\right)\right)$ while ensuring quick generalization to new tasks across different modalities (i.e. $\left.\left(y_{s}, y_{t}\right)\right)$. Wefi rst define encoders $e_{s}, e_{t}$ for source and target modalities (i.e., one specialized encoder for each modality) to map data or features from that modality to an aligned space. Meta-alignment trains encoders $e_{s}, e_{t}$ across multiple alignment tasks $\left\{\mathcal{T}_{a, 1}, \ldots, \mathcal{T}_{a, T}\right\}$ into an aligned space. Each alignment task $\mathcal{T}_{a}$ consists of paired data across source and target modalities. We explicitly train for generalization to new tasks by training metaalignment parameters $e_{s}^{\text {meta }}$ and $e_{t}^{\text {meta }}$ that are used to initialize instances of alignment models for new tasks [17]. When presented with a new task, wefi rst initialize task parameters using meta parameters $e_{s}:=e_{s}^{\text {meta }}, e_{t}:=e_{t}^{\text {meta }}$ before training on the task by optimizing for the NCE loss:

$\mathcal{L}_{\text {strong align }}=\sum_{\left(x_{s}, x_{t}\right) \in \mathcal{T}_{a}}\left(-e_{s}\left(x_{s}\right)^{\top} e_{t}\left(x_{t}\right)+\sum_{x_{t, \text { neg }}} e_{s}\left(x_{s}\right)^{\top} e_{t}\left(x_{t, \text { neg }}\right)\right)$. 


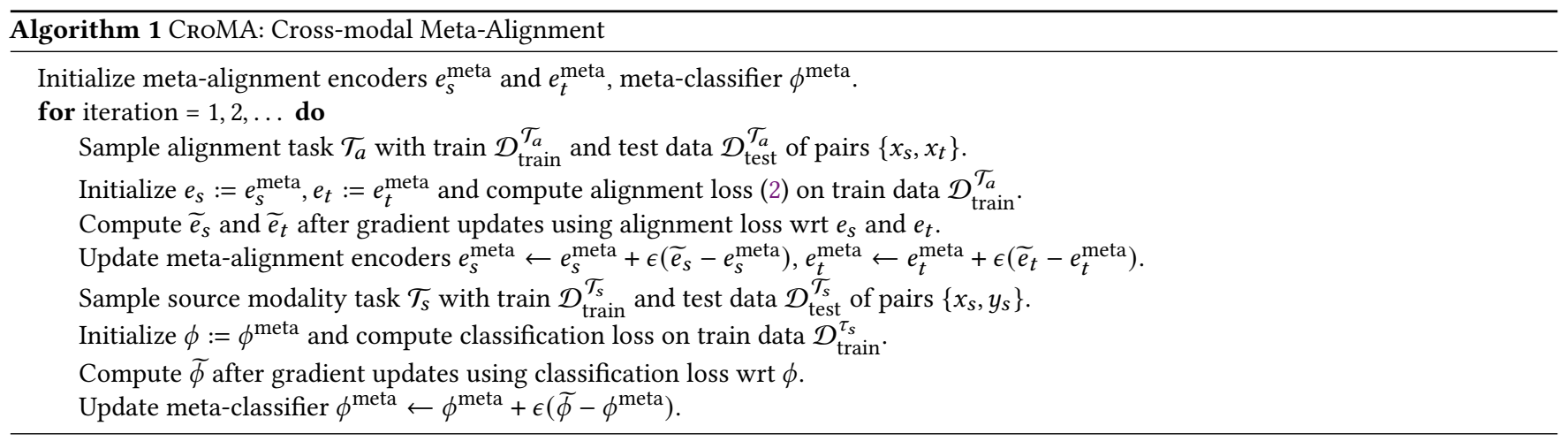

where $x_{t \text {,neg }}$ denotes unpaired negative samples. The NCE objective has a nice interpretation as capturing a space where the representations of similar concepts expressed in different modalities are close together, and different concepts are far apart [18, 47]. The metaparameters $e_{s}^{\text {meta }}$ and $e_{t}^{\text {meta }}$ are updated usingfi rst-order gradient information [45] so that they gradually become better initializations for new alignment tasks spanning new concepts.

4.1.2 Weak alignment. We now relax the data requirements from strong to weak alignment. Instead of one-to-one correspondences, weak alignment represent coarse groupings of semantic correspondence (see Figure 4(b)). This better reflects real-world multimodal data since cross-modal mappings are often many-to-many (e.g. many ways of describing an image, many ways of speaking the same sentence), and are abundant on the internet such as videos constituting weak pairs of image, audio, text [48, 67]. We denote a weak pair as sets $X_{s}$ and $X_{t}$, and define contrastive loss with an expectation over pairs across the sets, i.e. $x_{s}, x_{t} \in X_{s} \times X_{t}$ :

$\mathcal{L}_{\text {weak align }}=\sum_{\substack{\left(X_{s}, X_{t}\right) \\ \in \mathcal{T}_{a}}}\left(-\sum_{\substack{\left(x_{s}, x_{t}\right) \\ \in X_{s} \times X_{t}}} e_{s}\left(x_{s}\right)^{\top} e_{t}\left(x_{t}\right)+\sum_{x_{t, \text { neg }}} e_{s}\left(x_{s}\right)^{\top} e_{t}\left(x_{t, \text { neg }}\right)\right)$

and call this weak alignment. We sample several $x_{s} \in X_{s}$ and $x_{t} \in$ $X_{t}$ to treat as paired samples, and obtain negative pairs $x_{t, \text { neg }}$ by sampling outside of the paired sets.

\subsection{Cross-modal Generalization}

Given an aligned space, we now train a single classifier parametrized by a set of meta-parameters $\phi^{\text {meta }}$ on top of the aligned space for generalization across tasks $\left(y_{s}, y_{t}\right)$. The joint set of classification tasks consists of tasks $\left\{\mathcal{T}_{s, 1}, \ldots, \mathcal{T}_{s, T}\right\}$ in the source modality and tasks $\left\{\mathcal{T}_{t, 1}, \ldots, \mathcal{T}_{t, T}\right\}$ in the target (the total number of tasks $T$ need not be the same). When presented with a new task, wefi rst initialize the classifier using meta parameters $\phi:=\phi^{\text {meta }}$ before training on the task by optimizing for the cross-entropy loss. The metaparameters $\phi^{\text {meta }}$ are updated usingfi rst-order gradient information [45] towards better initialization parameters to classify new concepts. Overall, the meta-training stage consists of alignment tasks $\mathcal{T}_{a}$ and classification tasks in the source modality $\mathcal{T}_{s}$. The meta-testing stage presents tasks in the target modality $\mathcal{T}_{t}$. Each task consists of $k$ labeled pairs to simulate an episode of $k$-shot learning. We show the full training algorithm in Algorithm 1 and a visual diagram in Figure 4(c).

During testing, a task $\mathcal{T}_{t}$ is sampled in the target modality. We initialize a new model with the trained meta-alignment encoder $e_{t}^{\text {meta }}$ and meta-classifier $\phi^{\text {meta }}$, and perform gradient updates with the $k$ labeled samples in the target modality. Note that throughout the entire training process, only $k$ labeled samples in the target modality are presented to CROMA, which better reflects scarce target modalities where even labeled data for different tasks is difficult to obtain.

\section{EXPERIMENTS}

We test generalization from text to image, image to audio, and text to speech classification tasks. Anonymized code is included in the supplementary. Experimental details and additional results are included in Appendix B and C.

\subsection{Datasets and Tasks}

5.1.1 Text to Image dataset. We use the Yummly-28K dataset [43] which contains parallel text descriptions and images of recipes. We create classification labels from the metadata by concatenating the meal type and cuisine, yielding 44 distinct classes. The large number of recipes and shared concepts between text and image makes it an ideal testbed for cross-modal generalization. We used a ResNet pretrained on ImageNet [11] to encode the images, pretrained BERT encoder [12] for text, and a shared network for prediction.

5.1.2 Image to Audio dataset. We combine two large unimodal classification datasets over images (CIFAR-10 and CIFAR-100 [35]) and audio made by various objects (ESC-50 [49]) with partially related label spaces. This allows us to leverage complementary information from both modalities while testing on new concepts. To obtain weak pairs, we map similar classes between the datasets using similarities from WordNet [42] and text cooccurrence. This yields 17 clusters of weak pairs (Appendix B.2 lists all the clusters). We used a ResNet pretrained on ImageNet [11] to encode images and a CNN pretrained on AudioSet [19] to encode audio [36, 52].

5.1.3 Text to Speech dataset. We use the Wilderness dataset, a largescale multimodal dataset composed of parallel multilingual text and speech data [5]. We use a subset of 99 languages for language classification from text (source) and speech (target) individually. The tasks are split such as there is no overlap between the text and 
Table 2: Performance on text to image generalization on Yummly-28K (top), image to audio concept classification from CIFAR to ESC-50 (middle), and text to speech generalization on the Wilderness dataset (bottom). CroMA is on par and sometimes outperforms the oracle target modality meta-learning approach that has seen thousands of labeled target samples, and also outperforms existing unimodal, domain adaptation, and cross-modal baselines. \#Target (labels) denotes the number of target modality samples and labels used during meta-training.

\begin{tabular}{|c|c|c|c|c|c|c|}
\hline TASKT & YPEA & PPROACH1-S & нот5-S & нот10- & HOT & \#TARGET(LABELS) \\
\hline \multirow{6}{*}{$\begin{array}{c}\text { Text } \\
\text { (Yummly) } \\
\downarrow \\
\text { Image } \\
\text { (Yummly) }\end{array}$} & \multirow{2}{*}{ Unimodal } & Pre-training $[3,12]$ & $33.1 \pm 2.8$ & $36.4 \pm 3.5$ & $49.0 \pm 3.8$ & $0(0)$ \\
\hline & & Unsup. meta-learning [26] (reconstruct) & $37.4 \pm 0.6$ & $41.7 \pm 3.7$ & $49.0 \pm 1.0$ & $5131(0)$ \\
\hline & \multirow{3}{*}{ Cross-modal } & Align + Classify $[10,24,50,59,62]$ & $37.1 \pm 3.0$ & $40.0 \pm 2.7$ & $47.8 \pm 6.6$ & $5131(0)$ \\
\hline & & Align + Meta Classify [53] & $39.4 \pm 2.5$ & $40.0 \pm 2.3$ & $48.8 \pm 7.8$ & $5131(0)$ \\
\hline & & CROMA (ours) & $39.7 \pm 1.3$ & $47.1 \pm 3.3$ & $51.1 \pm 2.1$ & $5131(0)$ \\
\hline & Oracle & Within modality generalization $[17,45]$ & $38.9 \pm 2.1$ & $42.1 \pm 1.4$ & $47.9 \pm 5.6$ & $5131(5131)$ \\
\hline \multirow{7}{*}{$\begin{array}{c}\text { Image } \\
\text { (CIFAR) } \\
\downarrow \\
\text { Audio } \\
\text { (ESC-50) }\end{array}$} & \multirow{3}{*}{ Unimodal } & Pre-training $[3,12]$ & $44.2 \pm 0.8$ & $72.3 \pm 0.3$ & $77.4 \pm 1.7$ & $0(0)$ \\
\hline & & Unsup. meta-learning [26] (reconstruct) & $36.3 \pm 1.8$ & $67.3 \pm 0.9$ & $76.6 \pm 2.1$ & $920(0)$ \\
\hline & & Unsup. meta-learning [26] (weak labels) & $45.6 \pm 1.3$ & $74.2 \pm 0.3$ & $83.7 \pm 0.1$ & $920(0)$ \\
\hline & \multirow{3}{*}{ Cross-modal } & Align + Classify $[10,24,50,59,62]$ & $45.3 \pm 0.8$ & $73.9 \pm 2.1$ & $78.8 \pm 0.1$ & $920(0)$ \\
\hline & & Align + Meta Classify [53] & $47.2 \pm 0.3$ & $77.1 \pm 0.7$ & $80.4 \pm 0.0$ & $920(0)$ \\
\hline & & CROMA (ours) & $47.5 \pm 0.2$ & $85.9 \pm 0.7$ & $92.7 \pm 0.4$ & $920(0)$ \\
\hline & Oracle & Within modality generalization $[17,45]$ & $45.9 \pm 0.2$ & $89.3 \pm 0.4$ & $94.5 \pm 0.3$ & $920(920)$ \\
\hline \multirow{10}{*}{$\begin{array}{c}\text { Text } \\
\text { (Wilderness) } \\
\downarrow \\
\text { Speech } \\
\text { (Wilderness) }\end{array}$} & \multirow{2}{*}{ Unimodal } & Pre-training $[3,12]$ & $55.2 \pm 8.6$ & $73.1 \pm 3.4$ & $84.3 \pm 0.1$ & $0(0)$ \\
\hline & & Unsup. meta-learning [26] (reconstruct) & $61.5 \pm 4.4$ & $83.5 \pm 4.0$ & $88.5 \pm 2.1$ & $4395(0)$ \\
\hline & \multirow{4}{*}{$\begin{array}{l}\text { Domain } \\
\text { Adaptation }\end{array}$} & Shared [29] & $55.6 \pm 10.2$ & $75.2 \pm 8.4$ & $81.9 \pm 3.9$ & $4395(0)$ \\
\hline & & Shared + Align [31] & $59.7 \pm 7.6$ & $78.4 \pm 6.2$ & $84.3 \pm 1.5$ & $4395(0)$ \\
\hline & & Shared + Domain confusion [60] & $59.5 \pm 7.2$ & $76.3 \pm 9.4$ & $83.9 \pm 1.8$ & $4395(0)$ \\
\hline & & Shared + Target labels [30] & $57.3 \pm 9.3$ & $76.2 \pm 8.4$ & $84.0 \pm 1.9$ & $4395(4395)$ \\
\hline & \multirow{3}{*}{ Cross-modal } & Align + Classify $[10,24,50,59,62]$ & $61.1 \pm 6.0$ & $74.8 \pm 2.1$ & $86.2 \pm 0.7$ & $4395(0)$ \\
\hline & & Align + Meta Classify [53] & $65.6 \pm 6.1$ & $89.9 \pm 1.5$ & $93.0 \pm 0.5$ & $4395(0)$ \\
\hline & & CROMA (ours) & $67.9 \pm 6.6$ & $90.6 \pm 1.5$ & $93.2 \pm 0.2$ & $4395(0)$ \\
\hline & Oracle & Within modality generalization $[17,45]$ & $61.3 \pm 11.2$ & $77.0 \pm 0.3$ & $87.5 \pm 0.6$ & $4395(4395)$ \\
\hline
\end{tabular}

speech samples used for classification and the pairs seen for strong alignment. We use LSTMs to encode both text and speech data.

5.1.4 Metrics. We report few-shot $(k=1,5,10)$ classification accuracy in the target modality byfixing 8 evaluation tasks, each comprised of 5 unseen target concepts during meta-test. We compute accuracy across all 8 tasks and repeat experiments 10 times to report mean and standard deviations.

\subsubsection{Baselines. We compare with 4 broad sets of baselines:}

1) Unimodal baselines only use unlabeled data from the target modality during meta-training following our low-resource assumption. The simplest baseline ignores meta-training and just fine-tunes on the tasks in meta-test starting from a (supervised [3] or unsupervised [12]) pre-trained model. To better leverage unlabeled target modality data, we also compare with unsupervised meta-learning [26] which performs self-supervised learning via reconstruction or weak labels during meta-training (see Appen$\operatorname{dix}$ B.2).

2) We modify Domain Adaptation (DA) methods to verify that it is necessary to use separate encoders and perform explicit alignment: a) Shared shares all encoder layer for both modalities except a separate linear layer that maps data from the target modality's input dimension to the source [29, 59]. b) Shared + Align further adds our alignment loss (contrastive loss) on top of the encoded representations, in a manner similar to [31]. c) Shared + Domain confusion further adds a domain confusion loss on top of the encoded representations [60]. d) Shared + Target labels also uses target modality labels during meta-training, similar to supervised DA [30] (details in Appendix D.2).

3) Cross-modal: We adapt domain adaptation and meta-learning for cross-modal generalization under the following categories: a) Align + Classify which uses supervised alignment methods such as adversarial learning [59], cycle reconstruction [10, 24], or contrastive loss [62] to align input spaces from multiple domains before training a shared classifier [50]. b) Align + Meta Classify which learns a shared space using standard supervised alignment [18] before meta-learning a classifier [53], and c) CROMA which represents our full model of jointly training for generalization across alignment and classification tasks. Since all methods are agnostic to the alignment algorithm used, we use contrastive loss with negative sampling (Section 4.1) for fair comparison across all baselines.

4) Oracle: The ideal (but likely unrealistic) scenario where metatraining and meta-testing both have labeled data in the target modality. We use Reptile [45] for target modality meta-learning. Since there is the least domain shift, we expect this method to perform best but requires large amounts of labeled target data. 
Table 3: Language classification predictions (incorrect and correct) on low-resource speech samples after training on labeled text data. Despite seeing just 5 labeled speech samples, our method is able to accurately classify low-resource languages.

\begin{tabular}{l||cc}
\hline SPEECH( TEXT IN PARENTHESIS) & OrACLECRO & MA (OURS) \\
\hline (Beda Yesus agot gu ofa oida Bua buroru Didif ojgomu) & Russian & Meax \\
(Ido hai Timotiu natile hampai moula Aturana Musa) & Jamaican Patois & Badaic \\
(Mu habotu pa kali Mataoqu osolae vekoi Rau sari Mua kana pa kauru Nenemu gua) & Avokaya & Roviana \\
\hline
\end{tabular}
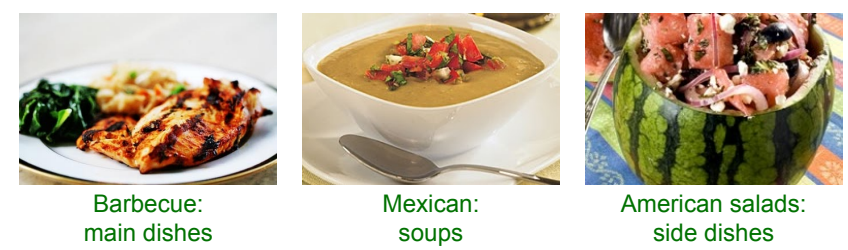

Figure 5: On Yummly-28K dataset, CroMA leverages source text to make accurate few-shot predictions on target images despite only seeing $1-10$ labeled image examples.

\subsection{Cross-modal Generalization}

5.2.1 Comparison to oracle. For text to image (Table 2 top) and text to speech (Table 2 bottom), CROMA surprisingly outperforms even the oracle baseline, in addition to unimodal and cross-modal methods. We hypothesize this is because text data (source) is cleaner than image and speech data (target) and the community has better models for encoding text than images and speech spectrograms. Consistent with this hypothesis, we found that text classifiers performed better on Yummly-28K and Wilderness datasets than image and speech classifiers. This implies that one can leverage abundant, cleaner, and more-predictive source modalities to improve target modality performance. For image to audio (Table 2 middle), we observe that our approach is on par (outperforms for $k=1$, and within $2-3 \%$ for $k=5,10$ ) with the oracle baseline that has seen a thousand labeled audio examples during meta-training.

5.2.2 Comparison to existing approaches. For all setups, CroMA consistently outperforms existing unimodal and cross-modal baselines. Since we use the same LSTM architecture for both text and speech, we can also apply DA approaches which share encoders. From Table 2 bottom, we see that they do not perform well on cross-modal generalization. Although domain confusion and alignment improve upon standard encoder sharing, they still fall short of our approach. Our method also outperforms the Shared + Target labels baseline which further uses target modality labels to train the shared encoder during meta-training. This serves to highlight the important differences between cross-modal generalization and DA: 1. separate encoders and 2. explicit alignment are important.

5.2.3 Ablation studies. Consistent across all setups in Table 2, we find that jointly meta-training across alignment and classification improves upon standard supervised alignment methods commonly used in domain adaptation $[50,53]$. Wefi nd that performance improvement is greatest for the 1-shot setting, suggesting that metaalignment is particularly suitable for low-resource target modalities.

5.2.4 Model predictions. We show samples of language classification on low-resource speech samples in Table 3. Despite seeing
Table 4: CroMA yields better alignment scores than the baselines, indicating that meta-alignment can align new concepts using only weakly paired data across image and audio.

\begin{tabular}{|c|c|c|c|c|c|c|}
\hline$K$ & EXPERIMENTR & @1 & $\mathrm{R} @ 5 \uparrow$ & R@10 & RANK $\downarrow$ & Cos. $\downarrow$ \\
\hline \multirow{3}{*}{5} & No align & $1.0 \%$ & $2.0 \%$ & $5.5 \%$ & 101 & 0.428 \\
\hline & Align & $2.0 \%$ & $5.5 \%$ & $8.5 \%$ & 103 & 0.272 \\
\hline & CroMA & $4.0 \%$ & $19.5 \%$ & $39.0 \%$ & 13 & 0.003 \\
\hline \multirow{3}{*}{10} & No align & $0.5 \%$ & $3.0 \%$ & $4.5 \%$ & 101 & 0.399 \\
\hline & Align & $1.5 \%$ & $11.0 \%$ & $18.5 \%$ & 52 & 0.222 \\
\hline & CROMA & $3.5 \%$ & $17.5 \%$ & $35.0 \%$ & 15 & 0.004 \\
\hline
\end{tabular}

just 5 labeled speech samples, our method is able to quickly generalize and classify low-resource languages. On text to image task (Figure 5), CroMA quickly recognizes images from new recipes.

\subsection{Few-shot Cross-modal Retrieval}

We show retrieval performance in Table 4 as measured using recall@k, rank, and cosine loss metrics [18]. Our model yields better retrieval performance than the baselines, indicating that meta-alignment successfully aligns new concepts in low-resource target modalities. In Figure 6, we also show samples of retrieved data in the target given input in the source modality to help us understand which source modalities the model is basing its target predictions on. Despite being trained only on weak pairs, meta-alignment is able to perform cross-modal retrieval atfi ne granularities.

\subsection{Noisy Target Labels}

We also evaluate the effect of noisy labels in the target modality since it is often difficult to obtain exact labels in low-resource modalities such as rare languages. We add symmetric noise [23] to all target modality labels (both meta-train and meta-test). Despite only seeing $k=1,5,10$ labels in the target, CROMA is more robust to noisy label than the oracle baseline (see Figure 7).

\subsection{On Alignment vs Supervision}

Finally, to study the tradeoffs in cross-modal alignment, we perform a controlled experiment on synthetic data from 2 modalities: source $D_{1}^{\text {sup }}=\left\{\left(x_{1}^{i}, y_{1}^{i}\right)\right\}_{i=1}^{n_{1}}$ and target $D_{2}^{\text {sup }}=\left\{\left(x_{2}^{i}, y_{2}^{i}\right)\right\}_{i=1}^{n_{2}}$. The labels are generated via a noisy teacher model $y_{m}^{i}=u_{m} x_{m}^{i}+\epsilon_{m}^{i}$, where $x_{m}^{i} \in \mathbb{R}^{d}, u_{m} \in \mathbb{R}^{d}$, and $\epsilon_{m}^{i} \sim \mathcal{N}\left(0, \sigma^{2}\right)$ for $m \in\{1,2\}$ [39]. We model cross-modal and task relationships through a full-rank transformation $x_{1}^{i}=W x_{2}^{i}$ and $u_{1} x_{1}^{i}=u_{2} W x_{2}^{i}$ respectively.

Suppose $n_{1} \gg n_{2}$ (i.e. high-resource source task with $n_{1}$ samples, low-resource target task with $n_{2}$ samples). One can train separate supervised models $f_{m}(x)=w_{m} x$ and measure the total generalization loss $\mathcal{L}=\sum_{m=1}^{2} \mathbb{E}_{x_{m}}\left[\left(f_{m}\left(x_{m}\right)-u_{m} x_{m}\right)^{2}\right]$, but this loss will be very high in the low-resource target task. Instead, 

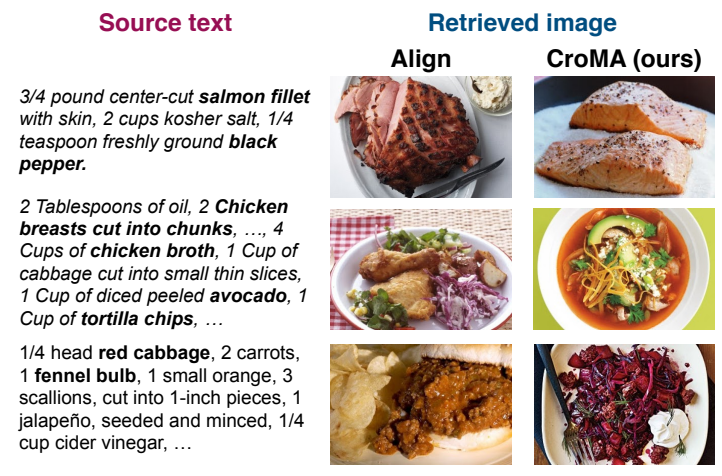

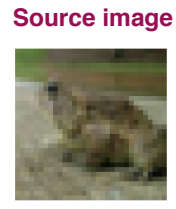

(Frog)

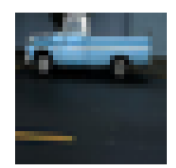

(Car)

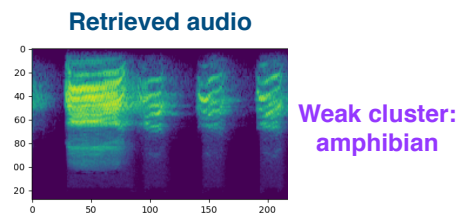

(Frog)

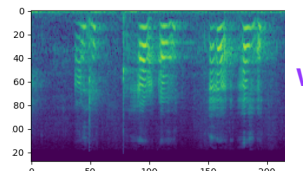

(Engine)

Weak cluster: automobile

Figure 6: Left: samples of retrieved images given text recipes. CROMA performs few-shot retrieval of images more accurately than existing alignment approaches. Right: samples of retrieved audio samples given images. Despite being trained only on weak pairs, meta-alignment can perform few-shot cross-modal retrieval atfi ne granularities (e.g. amphibian, automobile).

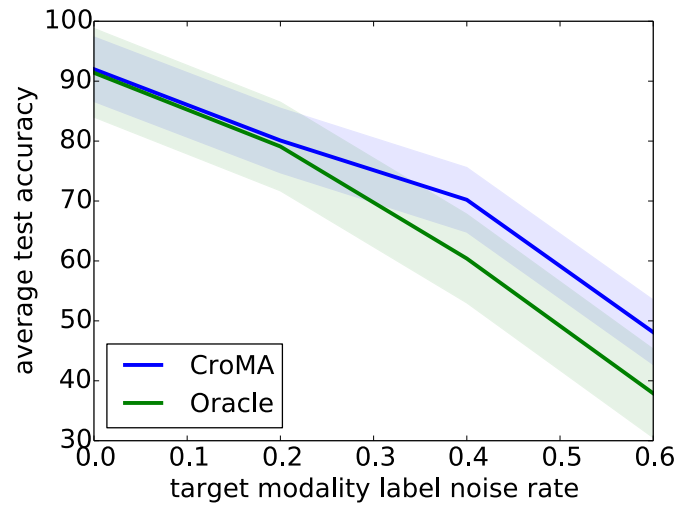

Figure 7: CroMA is robust to noisy labels in the target by using information from the source, making it suitable for low-resource modalities with imperfect annotations.

cross-modal alignment learns the transformation $W$ using pairs $D_{\text {unsup }}=\left\{\left(x_{1}^{i}, x_{2}^{i}\right)\right\}_{i=1}^{n_{\text {align }}}$ generated via $x_{1}^{i}=W x_{2}^{i}+\eta^{i}$ with noise $\eta^{i} \sim \mathcal{N}\left(0, \sigma_{W}^{2}\right) \cdot \eta^{i}$ models uncertainty in alignment pairs: $\sigma_{W}^{2} \rightarrow 0$ represents strong alignment and large $\sigma_{W}^{2}$ represents weak alignment.

We empirically study this setup in Figure 8, where we set $d=$ $20, n_{1}=250, n_{2}=40$ and vary $n_{\text {align }}$. We observe that (1) more alignment pairs help, but at most by the performance of the highresource source task, (2) quality of alignment matters: less noise $\sigma_{W}^{2}$ in alignment data gives better performance, and (3) even weak alignment is preferable to supervised learning with enough weakly paired data. In fact, under this simplified setup, an analysis shows that training on the high-resource task has error $d \sigma^{2} / n_{1}$ while the low-resource task has error $d \sigma^{2} / n_{2}$. Estimating the alignment matrix with $d^{2}$ elements results in error $d^{2} \sigma_{W}^{2} / n_{\text {align. Therefore, }}$ cross-modal alignment has error $\frac{d^{2} \sigma_{W}^{2}}{n_{\text {align }}}+\frac{d \sigma^{2}}{n_{1}}$, which should be preferred when $\frac{d \sigma_{W}^{2}}{n_{\text {align }}}+\frac{\sigma^{2}}{n_{1}}<\frac{\sigma^{2}}{n_{2}}$. This gives a simple rule-of-thumb for practitioners to choose between supervised learning and crossmodal learning (see Appendix E for more details and experiments).

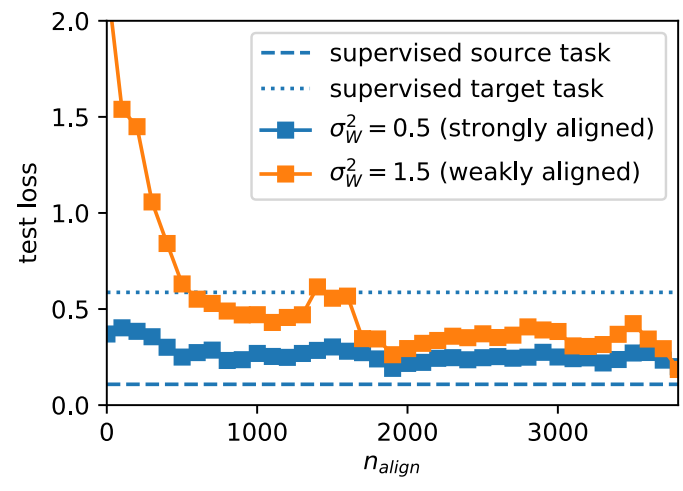

Figure 8: Supervision learning vs alignment for synthetic data: with more strongly or weakly aligned pairs $\left(n_{\text {align }}\right)$, cross-modal alignment improves upon supervised learning in target tasks.

\section{CONCLUSION}

In this work, we proposed cross-modal generalization: a learning paradigm where abundant source modalities are used to help lowresource target modalities. We showed that meta-alignment using cross-modal data can allow quick generalization to new concepts across different modalities. Our experiments demonstrate strong performance on classifying data from an entirely new target modality under limited samples and noisy labels, which is useful for generalization to low-resource images, speech, and languages.

\section{ACKNOWLEDGEMENTS}

This material is based upon work partially supported by the National Science Foundation (Awards \#1722822 and \#1750439), and National Institutes of Health (Awards \#R01MH125740, \#R01MH096951, \#U01MH116925, and \#U01MH116923). RS is supported in part by NSF IIS1763562 and ONR Grant N000141812861. Any opinions,findings, conclusions, or recommendations expressed in this material are those of the author(s) and do not necessarily reflect the views of the National Science Foundation, National Institutes of Health, and Office of Naval Research, and no official endorsement should be inferred. We would also like thank the anonymous reviewers for their feedback and NVIDIA's GPU support. 


\section{REFERENCES}

[1] Sami Abu-El-Haija, Nisarg Kothari, Joonseok Lee, Apostol (Paul) Natsev, George Toderici, Balakrishnan Varadarajan, and Sudheendra Vijayanarasimhan. 2016 YouTube-8M: A Large-Scale Video Classification Benchmark. In arXiv:1609.08675. https://arxiv.org/pdf/1609.08675v1.pdf

[2] Antreas Antoniou, Amos Storkey, and Harrison Edwards. 2017. Data augmentation generative adversarial networks. arXiv preprint arXiv:1711.04340 (2017).

[3] Alexei Baevski, Michael Auli, and Abdelrahman Mohamed. 2019. Effective ness of self-supervised pre-training for speech recognition. arXiv preprint arXiv:1911.03912 (2019)

[4] Tadas Baltrušaitis, Chaitanya Ahuja, and Louis-Philippe Morency. 2018. Multimodal machine learning: A survey and taxonomy. IEEE transactions on pattern analysis and machine intelligence 41, 2 (2018), 423-443.

[5] A. W. Black. 2019. CMU Wilderness Multilingual Speech Dataset. In ICASSP 2019 - 2019 IEEE International Conference on Acoustics, Speech and Signal Processing (ICASSP). 5971-5975.

[6] Zhangjie Cao, Mingsheng Long, Jianmin Wang, and Qiang Yang. 2017. Transitive hashing network for heterogeneous multimedia retrieval. In Proceedings of the Thirty-First AAAI Conference on Artificial Intelligence. 81-87.

[7] Rich Caruana. 1997. Multitask learning. Machine learning 28, 1 (1997), 41-75.

[8] Wei-Yu Chen, Yen-Cheng Liu, Zsolt Kira, Yu-Chiang Frank Wang, and JiaBin Huang. 2019. A closer look at few-shot classification. arXiv preprint arXiv:1904.04232 (2019)

[9] François Chollet. 2019. On the Measure of Intelligence. CoRR abs/1911.01547 (2019). arXiv:1911.01547 http://arxiv.org/abs/1911.01547

[10] Safa Cicek and Stefano Soatto. 2019. Unsupervised domain adaptation via regularized conditional alignment. In Proceedings of the IEEE International Conference on Computer Vision. 1416-1425.

[11] Jia Deng, Wei Dong, Richard Socher, Li-Jia Li, Kai Li, and Li Fei-Fei. 2009. Imagenet: A large-scale hierarchical image database. In 2009 IEEE conference on computer vision and pattern recognition. Ieee, 248-255.

[12] Jacob Devlin, Ming-Wei Chang, Kenton Lee, and Kristina Toutanova. 2018. Bert: Pre-training of deep bidirectional transformers for language understanding. arXiv preprint arXiv:1810.04805 (2018).

[13] Nanqing Dong and Eric P Xing. 2018. Domain adaption in one-shot learning In foint European Conference on Machine Learning and Knowledge Discovery in Databases. Springer, 573-588.

[14] Jared Dunnmon, Alexander Ratner, Nishith Khandwala, Khaled Saab, Matthew Markert, Hersh Sagreiya, Roger E. Goldman, Christopher Lee-Messer, Matthew P. Lungren, Daniel L. Rubin, and Christopher Ré. 2019. Cross-Modal Data Programming Enables Rapid Medical Machine Learning. CoRR abs/1903.11101 (2019) arXiv:1903.11101 http://arxiv.org/abs/1903.11101

[15] Chris Dyer. 2014. Notes on noise contrastive estimation and negative sampling. arXiv preprint arXiv:1410.8251 (2014)

[16] Fartash Faghri, David J Fleet, Jamie Ryan Kiros, and Sanja Fidler. 2017. Vse++ Improving visual-semantic embeddings with hard negatives. arXiv preprint arXiv:1707.05612 (2017)

[17] Chelsea Finn, Pieter Abbeel, and Sergey Levine. 2017. Model-agnostic metalearning for fast adaptation of deep networks. In Proceedings of the 34th International Conference on Machine Learning-Volume 70. JMLR. org, 1126-1135.

[18] Andrea Frome, Greg S Corrado, Jon Shlens, Samy Bengio, Jeff Dean, Marc'Aurelio Ranzato, and Tomas Mikolov. 2013. Devise: A deep visual-semantic embedding model. In Advances in neural information processing systems. 2121-2129.

[19] Jort F Gemmeke, Daniel PW Ellis, Dylan Freedman, Aren Jansen, Wade Lawrence, R Channing Moore, Manoj Plakal, and Marvin Ritter. 2017. Audio set: An ontology and human-labeled dataset for audio events. In 2017 IEEE International Conference on Acoustics, Speech and Signal Processing (ICASSP). IEEE, 776-780.

[20] Marzyeh Ghassemi, Tristan Naumann, Peter Schulam, Andrew L. Beam, and Rajesh Ranganath. 2018. Opportunities in Machine Learning for Healthcare. CoRR abs/1806.00388 (2018). arXiv:1806.00388 http://arxiv.org/abs/1806.00388

[21] Edouard Grave, Armand Joulin, and Quentin Berthet. 2018. Unsupervised Align ment of Embeddings with Wasserstein Procrustes. CoRR abs/1805.11222 (2018). arXiv:1805.11222 http://arxiv.org/abs/1805.11222

[22] Jiatao Gu, Hany Hassan, Jacob Devlin, and Victor O. K. Li. 2018. Universal Neural Machine Translation for Extremely Low Resource Languages. CoRR abs/1802.05368 (2018). arXiv:1802.05368 http://arxiv.org/abs/1802.05368

[23] Bo Han, Quanming Yao, Xingrui Yu, Gang Niu, Miao Xu, Weihua Hu, Ivor W. Tsang, and Masashi Sugiyama. 2018. Co-sampling: Training Robust Networks for Extremely Noisy Supervision. CoRR abs/1804.06872 (2018). arXiv:1804.06872 http://arxiv.org/abs/1804.06872

[24] Judy Hoffman, Eric Tzeng, Taesung Park, Jun-Yan Zhu, Phillip Isola, Kate Saenko, Alexei A. Efros, and Trevor Darrell. 2017. CyCADA: Cycle-Consistent Adversarial Domain Adaptation. CoRR abs/1711.03213 (2017). arXiv:1711.03213 http://arxiv org/abs/1711.03213

[25] Timothy Hospedales, Antreas Antoniou, Paul Micaelli, and Amos Storkey. 2020 Meta-learning in neural networks: A survey. arXiv preprint arXiv:2004.05439 (2020).
[26] Kyle Hsu, Sergey Levine, and Chelsea Finn. 2018. Unsupervised Learning via Meta-Learning. CoRR abs/1810.02334 (2018). arXiv:1810.02334 http://arxiv.org/ abs/1810.02334

[27] Yen-Chang Hsu, Zhaoyang Lv, and Zsolt Kira. 2017. Learning to cluster in order to transfer across domains and tasks. arXiv preprint arXiv:1711.10125 (2017).

[28] Xin Huang, Yuxin Peng, and Mingkuan Yuan. 2017. Cross-modal common representation learning by hybrid transfer network. In Proceedings of the 26th International foint Conference on Artificial Intelligence. 1893-1900.

[29] Mi-Young Huh, Pulkit Agrawal, and Alexei A. Efros. 2016. What makes ImageNet good for transfer learning? CoRR abs/1608.08614 (2016). arXiv:1608.08614 http: //arxiv.org/abs/1608.08614

[30] Hal Daumé III. 2009. Frustratingly Easy Domain Adaptation. CoRR abs/0907.1815 (2009). arXiv:0907.1815 http://arxiv.org/abs/0907.1815

[31] Pratik Jawanpuria, Mayank Meghwanshi, and Bamdev Mishra. 2020. Geometryaware Domain Adaptation for Unsupervised Alignment of Word Embeddings. arXiv preprint arXiv:2004.08243 (2020).

[32] Annervaz K M, Somnath Basu Roy Chowdhury, and Ambedkar Dukkipati. 2018. Learning beyond Datasets: Knowledge Graph Augmented Neural Networks for Natural Language Processing. In Proceedings of the 2018 Conference of the North American Chapter of the Association for Computational Linguistics: Human Language Technologies, Volume 1 (Long Papers).

[33] Mikhail Khodak, Maria-Florina Balcan, and Ameet Talwalkar. 2019. Provable Guarantees for Gradient-Based Meta-Learning. CoRR abs/1902.10644 (2019). arXiv:1902.10644 http://arxiv.org/abs/1902.10644

[34] Ranjay Krishna, Yuke Zhu, Oliver Groth, Justin Johnson, Kenji Hata, Joshua Kravitz, Stephanie Chen, Yannis Kalantidis, Li-Jia Li, David A. Shamma, Michael S. Bernstein, and Fei-Fei Li. 2016. Visual Genome: Connecting Language and Vision Using Crowdsourced Dense Image Annotations. CoRR abs/1602.07332 (2016). arXiv:1602.07332 http://arxiv.org/abs/1602.07332

[35] Alex Krizhevsky, Geoffrey Hinton, et al. 2009. Learning multiple layers of features from tiny images. (2009).

[36] Anurag Kumar, Maksim Khadkevich, and Christian Fugen. 2018. Knowledge Transfer from Weakly Labeled Audio Using Convolutional Neural Network for Sound Events and Scenes. In 2018 IEEE International Conference on Acoustics, Speech and Signal Processing (ICASSP). 326-330.

[37] Anders Boesen Lindbo Larsen, Søren Kaae Sønderby, Hugo Larochelle, and Ole Winther. 2016. Autoencoding beyond pixels using a learned similarity metric. In International conference on machine learning. PMLR, 1558-1566.

[38] Xinjian Li, Zhong Zhou, Siddharth Dalmia, Alan W Black, and Florian Metze. 2019. SANTLR: Speech Annotation Toolkit for Low Resource Languages. arXiv preprint arXiv:1908.01067 (2019)

[39] Paul Pu Liang, Terrance Liu, Liu Ziyin, Ruslan Salakhutdinov, and Louis-Philippe Morency. 2020. Think locally, act globally: Federated learning with local and global representations. arXiv preprint arXiv:2001.01523 (2020).

[40] Paul Pu Liang, Zhun Liu, Yao-Hung Hubert Tsai, Qibin Zhao, Ruslan Salakhutdinov, and Louis-Philippe Morency. 2019. Learning Representations from Imperfect Time Series Data via Tensor Rank Regularization. In Proceedings of the 57th Annual Meeting of the Association for Computational Linguistics. 1569-1576.

[41] Paul Pu Liang, Amir Zadeh, and Louis-Philippe Morency. 2018. Multimodal local-global ranking fusion for emotion recognition. In Proceedings of the 20th ACM International Conference on Multimodal Interaction. 472-476.

[42] George A. Miller. 1995. WordNet: A Lexical Database for English. Commun. ACM 38, 11 (Nov. 1995), 39-41.

[43] W. Min, S. Jiang, J. Sang, H. Wang, X. Liu, and L. Herranz. 2017. Being a Supercook: Joint Food Attributes and Multimodal Content Modeling for Recipe Retrieval and Exploration. IEEE Transactions on Multimedia 19, 5 (2017), 1100-1113. https: //doi.org/10.1109/TMM.2016.2639382

[44] Tsendsuren Munkhdalai and Hong Yu. 2017. Meta networks. In Proceedings of the 34th International Conference on Machine Learning-Volume 70. JMLR. org, 2554-2563.

[45] Alex Nichol and John Schulman. 2018. Reptile: a scalable metalearning algorithm. arXiv preprint arXiv:1803.02999 2 (2018), 2.

[46] OpenAI, Marcin Andrychowicz, Bowen Baker, Maciek Chociej, Rafal Józefowicz, Bob McGrew, Jakub W. Pachocki, Jakub Pachocki, Arthur Petron, Matthias Plappert, Glenn Powell, Alex Ray, Jonas Schneider, Szymon Sidor, Josh Tobin, Peter Welinder, Lilian Weng, and Wojciech Zaremba. 2018. Learning Dexterous In-Hand Manipulation. CoRR abs/1808.00177 (2018). arXiv:1808.00177 http://arxiv.org/abs/1808.00177

[47] Akila Pemasiri, Kien Nguyen, Sridha Sridharan, and Clinton Fookes. 2018. Semantic Correspondence: A Hierarchical Approach. CoRR abs/1806.03560 (2018). arXiv:1806.03560 http://arxiv.org/abs/1806.03560

[48] Veronica Perez-Rosas, Rada Mihalcea, and Louis-Philippe Morency. 2013. Utterance-Level Multimodal Sentiment Analysis. In Association for Computational Linguistics (ACL). Sofia, Bulgaria.

[49] Karol J Piczak. 2015. ESC: Dataset for environmental sound classification. In Proceedings of the 23rd ACM international conference on Multimedia. 1015-1018.

[50] Anant Raj, Vinay P. Namboodiri, and Tinne Tuytelaars. 2015. Subspace Alignment Based Domain Adaptation for RCNN Detector. CoRR abs/1507.05578 (2015). arXiv:1507.05578 http://arxiv.org/abs/1507.05578 
[51] Sachin Ravi and Hugo Larochelle. 2016. Optimization as a model for few-shot learning. (2016).

[52] Tal Ridnik, Hussam Lawen, Asaf Noy, and Itamar Friedman. 2020. TResNet: High Performance GPU-Dedicated Architecture. arXiv preprint arXiv:2003.13630 (2020).

[53] Doyen Sahoo, Hung Le, Chenghao Liu, and Steven C. H. Hoi. 2019. Meta-Learning with Domain Adaptation for Few-Shot Learning under Domain Shift. https //openreview.net/forum?id=ByGOuo0cYm

[54] Tal Schuster, Ori Ram, Regina Barzilay, and Amir Globerson. 2019. Cross-Lingual Alignment of Contextual Word Embeddings, with Applications to Zero-shot Dependency Parsing. CoRR abs/1902.09492 (2019). arXiv:1902.09492 http://arxiv. org/abs/1902.09492

[55] Jake Snell, Kevin Swersky, and Richard Zemel. 2017. Prototypical networks for few-shot learning. In Advances in neural information processing systems. 4077 4087.

[56] Richard Socher, Milind Ganjoo, Christopher D. Manning, and Andrew Y. Ng. 2013. Zero-Shot Learning through Cross-Modal Transfer. In Proceedings of the 26th International Conference on Neural Information Processing Systems - Volume 1 (Lake Tahoe, Nevada) (NIPS'13).

[57] Chuanqi Tan, Fuchun Sun, Tao Kong, Wenchang Zhang, Chao Yang, and Chunfang Liu. 2018. A Survey on Deep Transfer Learning. CoRR abs/1808.01974 (2018) arXiv:1808.01974 http://arxiv.org/abs/1808.01974

[58] Yao-Hung Hubert Tsai and Ruslan Salakhutdinov. 2017. Improving OneShot Learning through Fusing Side Information. CoRR abs/1710.08347 (2017) arXiv:1710.08347 http://arxiv.org/abs/1710.08347

[59] Eric Tzeng, Judy Hoffman, Kate Saenko, and Trevor Darrell. 2017. Adversarial discriminative domain adaptation. In Proceedings of the IEEE Conference on Computer Vision and Pattern Recognition. 7167-7176.

[60] Eric Tzeng, Judy Hoffman, Ning Zhang, Kate Saenko, and Trevor Darrell. 2014 Deep Domain Confusion: Maximizing for Domain Invariance. CoRR abs/1412.3474
(2014). arXiv:1412.3474 http://arxiv.org/abs/1412.3474

[61] Oriol Vinyals, Charles Blundell, Timothy Lillicrap, Daan Wierstra, et al. 2016. Matching networks for one shot learning. In Advances in neural information processing systems. $3630-3638$.

[62] Chang Wang and Sridhar Mahadevan. 2011. Heterogeneous Domain Adaptation Using Manifold Alignment. In Proceedings of the Twenty-Second International foint Conference on Artificial Intelligence - Volume Volume Two (Barcelona, Catalonia, Spain) (IfCAI'11). AAAI Press, 1541-1546.

[63] Zirui Wang, Jiateng Xie, Ruochen Xu, Yiming Yang, Graham Neubig, and Jaime Carbonell. 2019. Cross-lingual Alignment vs Joint Training: A Comparative Study and A Simple Unified Framework. arXiv preprint arXiv:1910.04708 (2019).

[64] Chen Xing, Negar Rostamzadeh, Boris Oreshkin, and Pedro O O. Pinheiro. 2019. Adaptive Cross-Modal Few-shot Learning. In Advances in Neural Information Processing Systems 32, H. Wallach, H. Larochelle, A. Beygelzimer, F. d'Alché-Buc, E. Fox, and R. Garnett (Eds.). http://papers.nips.cc/paper/8731-adaptive-crossmodal-few-shot-learning.pdf

[65] Zhenguo Yang, Min Cheng, Qing Li, Yukun Li, Zehang Lin, and Wenyin Liu. 2017. Cross-Domain and Cross-Modality Transfer Learning for Multi-domain and Multi-modality Event Detection. In Web Information Systems Engineering - WISE 2017, Athman Bouguettaya, Yunjun Gao, Andrey Klimenko, Lu Chen, Xiangliang Zhang, Fedor Dzerzhinskiy, Weijia Jia, Stanislav V. Klimenko, and Qing Li (Eds.). Springer International Publishing, Cham, 516-523.

[66] Amir Zadeh, Paul Pu Liang, and Louis-Philippe Morency. 2020. Foundations of Multimodal Co-learning. Information Fusion 64 (2020), 188-193.

[67] AmirAli Bagher Zadeh, Paul Pu Liang, Soujanya Poria, Erik Cambria, and LouisPhilippe Morency. 2018. Multimodal language analysis in the wild: Cmu-mosei dataset and interpretable dynamic fusion graph. In $A C L$.

[68] Qiming Zhang, Jing Zhang, Wei Liu, and Dacheng Tao. 2019. Category AnchorGuided Unsupervised Domain Adaptation for Semantic Segmentation. In Advances in Neural Information Processing Systems. 433-443. 